\title{
A RAMSEY-TYPE THEOREM FOR MULTIPLE DISJOINT COPIES OF INDUCED SUBGRAPHS
}

\author{
TOMOKI NAKAMIGAWA ${ }^{1}$ \\ Department of Information Science \\ Shonan Institute of Technology \\ 1-1-25 Tsujido-Nishikaigan, Fujisawa \\ Kanagawa 251-8511, Japan \\ e-mail: nakami@info.shonan-it.ac.jp
}

\begin{abstract}
Let $k$ and $\ell$ be positive integers with $\ell \leq k-2$. It is proved that there exists a positive integer $c$ depending on $k$ and $\ell$ such that every graph of order $(2 k-1-\ell / k) n+c$ contains $n$ vertex disjoint induced subgraphs, where these subgraphs are isomorphic to each other and they are isomorphic to one of four graphs: (1) a clique of order $k,(2)$ an independent set of order $k,(3)$ the join of a clique of order $\ell$ and an independent set of order $k-\ell$, or (4) the union of an independent set of order $\ell$ and a clique of order $k-\ell$.
\end{abstract}

Keywords: graph decomposition, induced subgraph, graph Ramsey theory, extremal graph theory.

2010 Mathematics Subject Classification: 05C55, 05C35.

\section{REFERENCES}

[1] S.A. Burr, On the Ramsey numbers $r(G, n H)$ and $r(n G, n H)$ when $n$ is large, Discrete Math. 65 (1987) 215-229. doi:10.1016/0012-365X(87)90053-7

[2] S.A. Burr, On Ramsey numbers for large disjoint unions of graphs, Discrete Math. 70 (1988) 277-293. doi:10.1016/0012-365X(88)90004-0

[3] S.A. Burr, P. Erdős and J.H. Spencer, Ramsey theorems for multiple copies of graphs, Trans. Amer. Math. Soc. 209 (1975) 87-99. doi:10.1090/S0002-9947-1975-0409255-0

\footnotetext{
${ }^{1}$ This work was supported by KAKENHI(23540168). The extended abstract of the paper is published in Electron. Notes Discrete Math. 43 (2013) 97-102, Erdős Centennial.
} 
[4] R.L. Graham, B.L. Rothschild and J.H. Spencer, Ramsey Theory, 2nd Edition (Wiley, New York, 1990).

[5] T. Nakamigawa, Vertex disjoint equivalent subgraphs of order 3, J. Graph Theory 56 (2007) 159-166.

doi:10.1002/jgt.20263

Received 22 May 2012

Revised 21 February 2013

Accepted 4 March 2013 